\title{
On generalized Humbert matrix polynomials
}

\author{
Levent Kargin and Veli Kurt
}




\title{
ON GENERALIZED HUMBERT MATRIX POLYNOMIALS
}

\author{
LEVENT KARGIN AND VELI KURT
}

Received 04 March, 2014

\begin{abstract}
Aktaş et. al. in [3] introduced the generalized Humbert matrix polynomials (G-HMP) $P_{n}^{A}(m, x, y, c)$. In this paper we focus on some properties of these matrix polynomials such as matrix recurrence relations, matrix differential equation and an integral representation. We introduce generalized forms of operational rules associated with operators corresponding to a G-HMP expansions. Moreover, we obtain a series transformation formula involving Gegenbauer matrix polynomials. Then we provide a number of applications.
\end{abstract}

2010 Mathematics Subject Classification: 33E20; 15A60; 35A22

Keywords: generalized Humbert matrix polynomials, Gegenbauer matrix polynomials, matrix differential equation

\section{INTRODUCTION}

Classical Humbert polynomials, which are generalized forms of Gegenbauer, Legendre, second kind Chebyshev, Pincherle polynomials, are defined in [16] and studied by many mathematicians. Through the last two decades, matrix polynomials have comprised an emerging field of study with important results in both theory and applications continuing to appear in the literature. Extension of the matrix framework of the classical families of polynomials are introduced [12,13,18,20,23,29] and several properties of the these matrix polynomials are established [4-6, 10,11,25,31,37]. Generalized Humbert matrix polynomials are introduced in [3]. Furthermore various families of bilinear and bilateral generating matrix functions and some relations of these matrix polynomials are presented [1,2]. By means of this study, choosing appropriate parameter we get both the known and the unknown properties of second kind Chebyshev, Legendre, Pincherle, Gegenbauer matrix polynomials which are introduced to date. The organization of this paper is as follows. In Section 2, starting from the generating matrix function, some matrix recurrence relations are given. Also, these polynomials appear as finite series solutions of $m t h$-order matrix differential equation are demonstrated. Section 3 deals with an integral representation and operational identities for G-HMP. Finally in Section 4, a series transformation formula for Gegenbauer matrix polynomials is established and some applications are given. 
Throughout this paper, the zero matrix and identity matrix will be denoted by 0 and $I$, respectively. If $A$ is a matrix in $\mathbb{C}^{r \times r}$, its spectrum $\sigma(A)$ denotes the set of all eigenvalues of $A$. Its 2-norm is denoted by $\|A\|$ and defined by

$$
\|A\|=\sup _{x \neq 0} \frac{\|A x\|_{2}}{\|x\|_{2}}
$$

where for a vector $y$ in $\mathbb{C}^{r},\|y\|_{2}=\left(y^{T}, y\right)^{\frac{1}{2}}$ is the Euclidean norm of $y$. If $f(z)$ and $g(z)$ are holomorphic functions of the complex variable $z$, which are defined in an open set $\Omega$ of the complex plane and $A$ is a matrix in $\mathbb{C}^{r \times r}$ such that $\sigma(A) \subset \Omega$, then from the properties of matrix functional calculus in [15, p. 558], it follows that $f(A) g(A)=g(A) f(A)$. For a matrix $A$ in $\mathbb{C}^{r \times r}$ the authors give the following relation due to [21]

$$
g(A)=(1-y)^{-A}=\sum_{n=0}^{\infty} \frac{(A)_{n}}{n !} y^{n}, \quad|y|<1,
$$

where $(A)_{n}$ is the Pochhammer symbol or shifted factorial which is defined by

$$
(A)_{n}=A(A+I)(A+2 I) \ldots(A+(n-1) I), \quad n \geq 1,
$$

with $(A)_{0}=I$ (see [21]). Moreover, from [22] for a matrix $A$ in $\mathbb{C}^{r \times r}$ such that $A+n I$ is invertible for every integer $n \geq 0$ we have

$$
(A)_{n}=\Gamma(A+n I) \Gamma^{-1}(A),
$$

where $\Gamma(A)$ is the gamma matrix function which is defined by [22]

$$
\Gamma(A)=\int_{0}^{\infty} e^{-t} t^{A-I} d t .
$$

Let $A$ be an arbitrary matrix in $\mathbb{C}^{r \times r}$. Then the Humbert matrix polynomials $P_{n}^{A}(m, x, y, c)$ are defined by generating matrix function

$$
\sum_{n=0}^{\infty} P_{n}^{A}(m, x, y, c) t^{n}=\left(c-m x t+y t^{m}\right)^{-A}=F(x, y, t, c, A),
$$

where $\left|m x t-y t^{m}\right|<|c|, m$ is a positive integer and the other parameters are unrestricted in general (see [3]). Also these matrix polynomials satisfy the following explicit expression

$$
P_{n}^{A}(m, x, y, c)=\sum_{k=0}^{\left[\frac{n}{m}\right]} \frac{(-1)^{k} c^{-(A+(n-m k+k) I)}(A)_{n-m k+k}}{k !(n-m k) !}(m x)^{n-m k} y^{k} .
$$

It is clear from (1.6) that

$$
P_{0}^{A}(m, x, y, c)=c^{-A}, \quad P_{1}^{A}(m, x, y, c)=\left\{\begin{array}{cc}
c^{-(A+I)} A m x & ; m>1 \\
c^{-(A+I)} A(x-y) & ; m=1
\end{array},\right.
$$




$$
P_{n}^{A}(m, x, y, c)=c^{-A} P_{n}^{A}\left(m, \frac{x}{c}, \frac{y}{c}\right),
$$

and

$$
\begin{aligned}
P_{n m}^{A}(m, 0, y, c) & =\frac{(-1)^{n} c^{-(A+n I)}(A)_{n} y^{n}}{n !}, \\
P_{n}^{A}(m, x, 0, c) & =\frac{c^{-(A+n I)}(A)_{n}(m x)^{n}}{n !} .
\end{aligned}
$$

For the special case of (1.6), we consider $P_{n}^{A}(m, x, y, c)$ as follows

$$
\begin{aligned}
& P_{n}^{A}(2, x, y, 1)=C_{n}^{A}(x, y), \\
& P_{n}^{[1]_{1 \times 1}}\left(2, x \sqrt{\frac{A}{2}}, y, 1\right)=U_{n}(x, y, A) \text {, } \\
& P_{n}^{\left[\frac{1}{2}\right]_{1 \times 1}}\left(2, x \sqrt{\frac{A}{2}}, 1,1\right)=P_{n}(x, A), \\
& P_{n}^{A}(3, x, 1,1)=h_{n}^{A}(x),
\end{aligned}
$$

where $C_{n}^{A}(x, y)$ : Gegenbauer matrix polynomials with two variables [19], $U_{n}(x, y, A)$ : second kind Chebyshev matrix polynomials with two variables [7], $P_{n}(x, A)$ : Legendre matrix polynomials [36], $h_{n}^{A}(x)$ : Pincherle matrix polynomials [27].

\section{RECURRENCE RELATIONS}

In this section we obtain matrix recurrence relations and $m t h$-order matrix differential equation for G-HMP.

Let us get some matrix recurrence relations for G-HMP. Differentiating (1.5) with respect to $c, x, y, t$ we have

$$
\begin{aligned}
\frac{\partial}{\partial c} F(x, y, t, c, A) & =\frac{-A}{c-m x t+y t^{m}} F(x, y, t, c, A), \\
\frac{\partial}{\partial x} F(x, y, t, c, A) & =\frac{t}{c-m x t+y t^{m}} m A F(x, y, t, c, A), \\
\frac{\partial}{\partial y} F(x, y, t, c, A) & =\frac{-t^{m}}{c-m x t+y t^{m}} A F(x, y, t, c, A), \\
\frac{\partial}{\partial t} F(x, y, t, c, A) & =\frac{\left(x-y t^{m-1}\right)}{c-m x t+y t^{m}} m A F(x, y, t, c, A) .
\end{aligned}
$$

We can rewrite (2.2) and (2.3) in the from

$$
m A\left(c-m x t+y t^{m}\right)^{-(A+I)}=\sum_{n=0}^{\infty} \frac{\partial}{\partial x} P_{n+1}^{A}(m, x, y, c) t^{n},
$$


and

$$
-A\left(c-m x t+y t^{m}\right)^{-(A+I)}=\sum_{n=0}^{\infty} \frac{\partial}{\partial y} P_{n+m}^{A}(m, x, y, c) t^{n},
$$

respectively. Applying (1.5) to the left-hand sides of equations (2.5) and (2.6) we have

$$
\begin{aligned}
\sum_{n=0}^{\infty} m A P_{n}^{A+I}(m, x, y, c) t^{n} & =\sum_{n=0}^{\infty} \frac{\partial}{\partial x} P_{n+1}^{A}(m, x, y, c) t^{n}, \\
\sum_{n=0}^{\infty}-A P_{n}^{A+I}(m, x, y, c) t^{n} & =\sum_{n=0}^{\infty} \frac{\partial}{\partial y} P_{n+m}^{A}(m, x, y, c) t^{n} .
\end{aligned}
$$

Comparing the coefficients of $t^{n}$ in the above equations we arrive at

$$
\begin{aligned}
& \frac{\partial}{\partial x} P_{n}^{A}(m, x, y, c)=m A P_{n-1}^{A+I}(m, x, y, c), \quad n \geq 1, \\
& \frac{\partial}{\partial y} P_{n}^{A}(m, x, y, c)=-A P_{n-m}^{A+I}(m, x, y, c), \quad n \geq m
\end{aligned}
$$

By iteration we obtain two differential relations for G-HMP

$$
\begin{aligned}
& \frac{\partial^{r}}{\partial x^{r}} P_{n}^{A}(m, x, y, c)=m^{r}(A)_{r} P_{n-r}^{A+r I}(m, x, y, c), \\
& \frac{\partial^{r}}{\partial y^{r}} P_{n}^{A}(m, x, y, c)=(-1)^{r}(A)_{r} P_{n-m r}^{A+r I}(m, x, y, c) .
\end{aligned}
$$

Therefore, we have

$$
\frac{\partial^{r}}{\partial x^{r}} P_{n}^{A}(m, x, y, c)+(-1)^{r-1} m^{r} \frac{\partial^{r}}{\partial y^{r}} P_{n+(m-1) r}^{A}(m, x, y, c)=\mathbf{0} .
$$

Considering equations (2.1) and (2.2), and applying the same method we get

$$
\frac{\partial^{r}}{\partial x^{r}} P_{n}^{A}(m, x, y, c)=(-1)^{r} m^{r} \frac{\partial^{r}}{\partial c^{r}} P_{n-r}^{A}(m, x, y, c), \quad n \geq r .
$$

Also, from equations (2.1) and (2.3) we have

$$
\frac{\partial^{r}}{\partial y^{r}} P_{n}^{A}(m, x, y, c)=\frac{\partial^{r}}{\partial c^{r}} P_{n-m r}^{A}(m, x, y, c), \quad n \geq m r .
$$

Let us consider the equations (2.2) and (2.4). Then we have

$$
\left(x-y t^{m-1}\right) \frac{\partial}{\partial x} F(x, y, t, c, A)-t \frac{\partial}{\partial t} F(x, y, t, c, A)=\mathbf{0} .
$$

Using (1.5) we get

$$
\sum_{n=0}^{\infty} x \frac{\partial}{\partial x} P_{n}^{A}(m, x, y, c) t^{n}-\sum_{n=0}^{\infty} y \frac{\partial}{\partial x} P_{n}^{A}(m, x, y, c) t^{n+m-1}
$$




$$
=\sum_{n=1}^{\infty} n P_{n}^{A}(m, x, y, c) t^{n} .
$$

Since $\frac{\partial}{\partial x} P_{0}^{A}(m, x, y, c)=0$, comparing the coefficients of $t^{n}$ yields

$$
x \frac{\partial}{\partial x} P_{n}^{A}(m, x, y, c)-n P_{n}^{A}(m, x, y, c)=y \frac{\partial}{\partial x} P_{n+1-m}^{A}(m, x, y, c) .
$$

Furthermore, from equations (2.2) and (2.4), we get

$$
\frac{m A\left(c-m x t+y t^{m}\right)^{-A}}{c-m x t+y t^{m}}=\sum_{n=0}^{\infty} \frac{\partial}{\partial x} P_{n}^{A}(m, x, y, c) t^{n-1}
$$

and

$$
\frac{\left(x-y t^{m-1}\right) m A\left(c-m x t+y t^{m}\right)^{-A}}{\left(c-m x t+y t^{m}\right)}=\sum_{n=1}^{\infty} n P_{n}^{A}(m, x, y, c) t^{n-1},
$$

respectively. Thus, multiplying (2.14) by $1-y(m-1) t^{m}$ and (2.15) by $m t$ and substracting them we get

$$
\begin{aligned}
y(m-1) \frac{\partial}{\partial x} P_{n+1-m}^{A} & (m, x, y, c) \\
= & \frac{\partial}{\partial x} P_{n+1}^{A}(m, x, y, c)-m(A+n I) P_{n}^{A}(m, x, y, c) .
\end{aligned}
$$

Using (2.13) in the above equation we have

$$
\frac{\partial}{\partial x} P_{n+1}^{A}(m, x, y, c)=(m-1) x \frac{\partial}{\partial x} P_{n}^{A}(m, x, y, c)+(m A+n I) P_{n}^{A}(m, x, y, c) .
$$

Finally, applying (2.7) in equations (2.16) and (2.17) respectively we obtain two more matrix recurrence relations

$$
\begin{aligned}
& m(A+n I) P_{n}^{A}(m, x, y, c) \\
& \quad=m A P_{n}^{A+I}(m, x, y, c)-m(m-1) A y P_{n-m}^{A+I}(m, x, y, c), \quad n \geq m \\
& \quad(m A+n I) P_{n}^{A}(m, x, y, c) \\
& \quad=m A P_{n}^{A+I}(m, x, y, c)-m(m-1) A x P_{n-1}^{A+I}(m, x, y, c), \quad n \geq 1 .
\end{aligned}
$$

From (2.4) we get

$$
\left(c-m x t+y t^{m}\right) \frac{\partial}{\partial t} F(x, y, t, c, A)=\left(x-y t^{m-1}\right) m A F(x, y, t, c, A) .
$$

Using (1.5) in the above equation we obtain

$$
\sum_{n=1}^{\infty}\left(c-m x t+y t^{m}\right) n P_{n}^{A}(m, x, y, c) t^{n-1}
$$




$$
=\sum_{n=0}^{\infty}\left(x-y t^{m-1}\right) m A P_{n}^{A}(m, x, y, c) t^{n} .
$$

By making appropriate changes of indices and comparing the coefficients of $t^{n}$, we obtain a three-term matrix recurrence relation for G-HMP

$$
\begin{aligned}
c(n+1) & P_{n+1}^{A}(m, x, y, c)-m x(A+n I) P_{n}^{A}(m, x, y, c) \\
& +y[m A+(n-m+1) I] P_{n+1-m}^{A}(m, x, y, c)=\mathbf{0}, \quad n \geq m-1 .
\end{aligned}
$$

Summary of these results is given in the following theorem.

Theorem 1. Let $A$ be an arbitrary matrix in $\mathbb{C}^{r \times r}$ and $m$ be a positive integer. Then G-HMP satisfy the differential relation (2.10) and recurrence relations (2.18), (2.19) and (2.20).

Before concluding this section we discuss the matrix differential equation of GHMP. Let the sequence $\left(f_{r}\right)_{r=0}^{n}$ be given by $f_{r}=f(r)$, where

$$
f(t)=(n-t)\left(\frac{(n-t) I+m(A+t I)}{m}\right)_{m-1} .
$$

Also we introduce two standard difference operators: the forward difference operator $\Delta$ and the displacement (or shift) operator $E$ are given as

$$
\Delta f_{r}=f_{r+1}-f_{r} \text { and } E f_{r}=f_{r+1}
$$

and their powers are given by

$$
\Delta^{0} f_{r}=f_{r}, \quad \Delta^{k} f_{r}=\Delta\left(\Delta^{k-1} f_{r}\right), \quad E^{k} f_{r}=f_{r+k} .
$$

Theorem 2. Let $A$ be an arbitrary matrix in $\mathbb{C}^{r \times r}$ and $m$ be a positive integer. Then $G-H M P$ are a particular solution of the $m$ th-order matrix differential equation in the form:

$$
c^{m-1} y \frac{\partial^{m}}{\partial x^{m}} P_{n}^{A}(m, x, y, c)+\sum_{s=0}^{m} a_{s} x^{s} \frac{\partial^{s}}{\partial x^{s}} P_{n}^{A}(m, x, y, c)=\mathbf{0} .
$$

where the coefficients $a_{s}$ are given by

$$
a_{s}=\frac{m^{m-1}}{s !} \Delta^{s} f_{0}
$$

Proof. Let $n=m p+q$, where $p=\left[\frac{n}{m}\right]$ and $0 \leq q \leq m-1$. Differentiating (1.6) with respect to $x$, we get

$x^{s} \frac{\partial^{s}}{\partial x^{s}} P_{n}^{A}(m, x, y, c)=\sum_{k=0}^{\left[\frac{n-s}{m}\right]} \frac{(-1)^{k} c^{-(A+(n-m k+k) I)}(A)_{n-(m-1) k}}{k !(n-m k-s) !}(m)^{n-m k} y^{k}$, and 


$$
\begin{aligned}
\frac{\partial^{m}}{\partial x^{m}} P_{n}^{A}(m, x, y, c) & \\
& =\sum_{k=0}^{p-1} \frac{(-1)^{k} c^{-(A+(n-m k+k) I)}(A)_{n-(m-1) k} m^{m}}{k !(n-m(k+1)) !}(m x)^{n-m(k+1)} y^{k},
\end{aligned}
$$

where $\left[\frac{n-s}{m}\right]=\left\{\begin{array}{cc}p & ; s \leq q \\ p-1 & ; s>q\end{array}\right.$. Substituting these expressions in the differential equation (2.21) and comparing the corresponding coefficients, we obtain

$$
\sum_{s=0}^{m}\left(\begin{array}{c}
n-m k \\
s
\end{array}\right) s ! a_{s}=m^{m} k(A+(n-(m-1) k) I)_{m-1}
$$

where $k=0,1,2, \cdots p-1$, and

$$
\sum_{s=0}^{q}\left(\begin{array}{c}
n-m p \\
s
\end{array}\right) s ! a_{s}=m^{m} p(A+(n-(m-1) k) I)_{m-1} .
$$

(2.24) can be written as

$$
\sum_{s=0}^{q}\left(\begin{array}{l}
q \\
s
\end{array}\right) m^{m-1} \Delta^{s} f_{0}=m^{m-1}(n-q)\left(A+\left(q+\frac{n-q}{m}\right) I\right)_{m-1} .
$$

This equality holds because it is equivalent to

$$
(1+\Delta)^{q} f_{0}=E^{q} f_{0}=f_{q}=f(q) .
$$

For $k=0,1,2, \cdots p-1,(2.23)$ can be written in the form

$$
\sum_{s=0}^{m}\left(\begin{array}{c}
n-m k \\
s
\end{array}\right) \Delta^{s} f_{0}=f_{n-m k} .
$$

Since $f(t)$ is a polynomial of degree $m$, the last equality holds and (2.25) is a forward difference formula for $f$ at the point $t=n-m k$. Thus, the proof is completed.

Corollary 1. Pincherle matrix polynomials are a solution of the third-order matrix differential equation in the form:

$$
\begin{aligned}
( & \left.1-\frac{32}{27} x^{3}\right) \frac{d^{3}}{d x^{3}} h_{n}^{A}(x)-\frac{16}{9}(2 A+3 I) x^{2} \frac{d^{2}}{d x^{2}} h_{n}^{A}(x) \\
& -\frac{8}{27}(3 n(2 A+(n+1) I)-(3 A+2 I)(3 A+5 I)) x \frac{d}{d x} h_{n}^{A}(x) \\
& +\frac{8}{27} n(3 A+n I)(3 A+(n+1) I) h_{n}^{A}(x)=\mathbf{0} .
\end{aligned}
$$




\section{An INTEgRAL REPRESENTATION AND Its APPLICATIONS}

Firstly, let us discuss the extension of classical Lahiri generalized Hermite polynomials (G-LHP) which are specified by the series definition

$$
H_{n, m}(x, y)=n ! \sum_{k=0}^{\left[\frac{n}{m}\right]} \frac{(-1)^{k} y^{k}(m x)^{n-m k}}{k !(n-m k) !}
$$

and by the generating function

$$
\sum_{n=0}^{\infty} H_{n, m}(x, y) \frac{t^{n}}{n !}=\exp \left(m x t-y t^{m}\right) .
$$

It is easy to show that

$$
H_{n, m}(x, y)=y^{\frac{n}{m}} H_{n}\left(\frac{x}{\sqrt[m]{y}}\right) \text { and } H_{n, m}(x, 1)=H_{n, m}(x),
$$

where $H_{n, m}(x)$ is the G-LHP given in [30]. In particular, for $m=2, H_{n, 2}(x, 1)=$ $H_{n}(x)$, where $H_{n}(x)$ denotes the ordinary Hermite polynomials [35]. Moreover, $H_{n, m}(x, y)=g_{n}^{m}(m x,-y)$, where $g_{n}^{m}(x, y)$ is the Gould-Hopper generalized Hermite polynomials (G-HGHP) [17].

Using the summation formula for G-HGHP in [28, Theorem 2.1 p. 1538] G-LHP satisfy

$$
H_{n+s, m}(w, y)=\sum_{k, r=0}^{n, s}\left(\begin{array}{l}
n \\
k
\end{array}\right)\left(\begin{array}{l}
s \\
r
\end{array}\right) m^{k+r}(w-x)^{k+r} H_{n+s-k-r, m}(x, y) .
$$

In the following theorem we give an integral representation for G-HMP including G-LHP.

Theorem 3. Let $A$ be a matrix in $\mathbb{C}^{r \times r}$ such that $\operatorname{Re}(z)>0$ for all $z \in \sigma(A), m$ be a positive integer and $c$ be a complex number such that $\operatorname{Re}(c)>0$. Then $G-H M P$ have the integral representation

$$
P_{n}^{A}(m, x, y, c)=\frac{\Gamma^{-1}(A)}{n !} \int_{0}^{\infty} e^{-c t} t^{A+(n-1) I} H_{n, m}\left(x, \frac{y}{t^{m-1}}\right) d t .
$$

Proof. Using (3.1) in the right-hand side of (3.4) we have

$$
\begin{aligned}
& \frac{\Gamma^{-1}(A)}{n !} \int_{0}^{\infty} e^{-c t} t^{A+(n-1) I} H_{n, m}\left(x, \frac{y}{t^{m-1}}\right) d t \\
& =\sum_{k=0}^{\left[\frac{n}{m}\right]} \frac{(-1)^{k} c^{-(A+(n-m k+k) I)}(m x)^{n-m k} y^{k}}{k !(n-m k) !} \Gamma^{-1}(A) \Gamma(A+(n-m k+k)) .
\end{aligned}
$$

From (1.3) we get the proof. 
As an application of this theorem we give the following proposition for G-HMP.

Proposition 1. G-HMP satisfy the summation formula

$$
P_{n+s}^{A}(m, w, y, c)=\sum_{k, r=0}^{n, s} \frac{\left(\begin{array}{l}
n \\
k
\end{array}\right)\left(\begin{array}{l}
s \\
r
\end{array}\right)(A)_{k+r} m^{k+r}(w-x)^{k+r}}{\left(\begin{array}{l}
n+s \\
k+r
\end{array}\right)(k+r) !} P_{n+s-k-r}^{A+(k+r) I}(m, x, y, c) .
$$

Proof. Replacing $n$ with $n+s$ in (3.4) and using the summation formula (3.3) we deduce

$$
\begin{array}{r}
P_{n+s}^{A}(m, w, y, c)=\sum_{k, r=0}^{n, s} \frac{(-1)^{k+r}\left(\begin{array}{l}
n \\
k
\end{array}\right)\left(\begin{array}{l}
s \\
r
\end{array}\right)(n+s-k-r) ! m^{k+r}(w-x)^{k+r}}{(n+s) !} \\
\frac{\partial^{k+r}}{\partial c^{k+r}} P_{n+s-k-r}^{A}(m, x, y, c) .
\end{array}
$$

From (2.8) we obtain the result.

Taking $k=0$ in assertion (3.5) of Proposition 1, we deduce the following consequence of Proposition 1.

Corollary 2. The following summation formula for the G-HMP holds

$$
P_{n}^{A}(m, w, y, c)=\sum_{k=0}^{n} \frac{(A)_{k} m^{k}(w-x)^{k}}{k !} P_{n-k}^{A+k I}(m, x, y, c) .
$$

Remark 1. Replacing $w$ by $w+x$ in (3.6), we obtain

$$
P_{n}^{A}(m, x+w, y, c)=\sum_{k=0}^{n} \frac{(A)_{k}(m w)^{k}}{k !} P_{n-k}^{A+k I}(m, x, y, c) .
$$

Now, we try to understand more deeply the role played by the integral transform connecting G-LHP and G-HMP. It is obvious that both $H_{n, m}(x, y)$ and $P_{n}^{A}(m, x, y, c)$ reduce to ordinary form for $y=1$.

It is easy to find that

$$
P_{n}^{A}(m, x, c)=\frac{\Gamma^{-1}(A)}{n !} \int_{0}^{\infty} e^{-c t} t^{A+\left(\frac{n}{m}-1\right) I} H_{n, m}\left(\frac{x t}{\sqrt[m]{t}}\right) d t .
$$

Using the fact that

$$
\exp \left(\lambda x \frac{d}{d x}\right) f(x)=f(x \exp \lambda)
$$

where $f(x)$ is an appropriate function, we have

$$
P_{n}^{A}(m, x, c)=\frac{\Gamma^{-1}(A)}{n !} \int_{0}^{\infty} e^{-c t} t^{A+\left(\frac{n}{m}+\left(1-\frac{1}{m}\right) x \frac{d}{d x}-1\right) I} d t H_{n, m}(x) .
$$

From (1.4) we acquire the following theorem. 
Theorem 4. Let $A$ be a matrix in $\mathbb{C}^{r \times r}$ such that $\operatorname{Re}(z)>0$ for all $z \in \sigma(A), m$ be a positive integer and $c$ be a complex number such that $\operatorname{Re}(c)>0$. Then $G-H M P$ satisfy

$$
\Gamma(A) n ! P_{n}^{A}(m, x, c)=\exp (-\widehat{Q} \ln c) \Gamma(\widehat{Q}) H_{n, m}(x),
$$

where $\widehat{Q}=\left[A+\left(\frac{n}{m}+\left(1-\frac{1}{m}\right) x \frac{d}{d x}\right) I\right]$.

We conclude this section giving another representation for the G-HMP.

Theorem 5. Let $A$ be an arbitrary matrix in $\mathbb{C}^{r \times r}$ and $m$ be a positive integer. Then G-HMP satisfy the identity

$$
P_{n}^{A}(m, x, y, c)=\frac{(A)_{n}}{n !} \exp \left[y(-m)^{-m} \widehat{D}_{c}^{-(m-1)} \frac{\partial^{m}}{\partial x^{m}}\right] c^{-(A+n I)}(m x)^{n},
$$

where $\widehat{D}_{c}^{-1}$ denotes the inverse derivative operator (see [14] for details).

Proof. Identities (2.11) and (2.12) allow us to conclude that

$$
(-m)^{m} \frac{\partial^{m}}{\partial c^{m-1} \partial y} P_{n}^{A}(m, x, y, c)=\frac{\partial^{m}}{\partial x^{m}} P_{n}^{A}(m, x, y, c) .
$$

The last identity and the fact that

$$
P_{n}^{A}(m, x, 0, c)=\frac{(A)_{n}}{n !} c^{-(A+n I)}(m x)^{n},
$$

give the proof.

We note that taking $A=[1]_{1 \times 1}, m=2$ and replacing $x I$ by $x \sqrt{\frac{A}{2}}$, the expressions (3.8) and (3.9) coincide with the formulas which was given in [24] for the second kind Chebyshev matrix polynomials.

\section{Some Results for Gegenbauer Matrix Polynomials}

In this section we deal with the integral representation for Gegenbauer matrix polynomials including classical Hermite polynomials. Using this representation we give a generating function of Gegenbauer matrix polynomials with two-variables. As a main theorem of this section we obtain a series transformation formula involving Gegenbauer matrix polynomials. We then provide a number of applications using appropriate binomial transformations.

Taking $c=1, m=2$ and replacing $n$ with $n+p$ in (3.4) we deduce

$$
C_{n+p}^{A}(x, y)=\frac{\Gamma^{-1}(A)}{(n+p) !} \int_{0}^{\infty} e^{-t} t^{A+(n+p-1) I} H_{n+p}\left(x, \frac{y}{t}\right) d t,
$$

Multiplying both hand sides of above equation by $\frac{(n+p) !}{n ! p !} z^{n}$ and summing over $n$ from zero to infinity, we get 


$$
\begin{aligned}
\sum_{n=0}^{\infty} \frac{(n+p) !}{n ! p !} C_{n+p}^{A}( & (x, y) z^{n} \\
& =\frac{\Gamma^{-1}(A)}{p !} \int_{0}^{\infty} e^{-t} t^{A+(p-1) I} \sum_{n=0}^{\infty} H_{n+p}\left(x, \frac{y}{t}\right) \frac{(z t)^{n}}{n !} d t
\end{aligned}
$$

Using identity [32]

$$
\sum_{n=0}^{\infty} H_{n+p}(x, y) \frac{z^{n}}{n !}=\exp \left(2 x z-y z^{2}\right) H_{p}(x-y z, y),
$$

we have

$$
\begin{aligned}
\sum_{n=0}^{\infty} \frac{(n+p) !}{n ! p !} C_{n+p}^{A}(x, y) z^{n} & \\
= & \frac{\Gamma^{-1}(A)}{p !} \int_{0}^{\infty} e^{-t\left(1-2 x z+y z^{2}\right) t^{A+(p-1) I}} H_{p}\left(x-y z, \frac{y}{t}\right) d t .
\end{aligned}
$$

This gives the following theorem for Gegenbauer matrix polynomials with twovariables.

Theorem 6. Let $A$ be a matrix in $\mathbb{C}^{r \times r}$ such that $\operatorname{Re}(z)>0$ for all $z \in \sigma(A), p$ be a positive integer. Then Gegenbauer matrix polynomials with two-variables have the generating function

$$
\begin{aligned}
\sum_{n=0}^{\infty}\left(\begin{array}{c}
n+p \\
p
\end{array}\right) & C_{n+p}^{A}(x, y) z^{n} \\
& =\left(1-2 x z+y z^{2}\right)^{-(A+p I)} C_{p}^{A}\left(x-y z,\left(1-2 x z+y z^{2}\right) y\right)
\end{aligned}
$$

where $\left|2 x z-y z^{2}\right|<1$.

We note that for the case $y=1$ the expression (4.2) coincides with the formula which was proved by Khammash ([26]) for the Gegenbauer matrix polynomials.

Before giving the main theorem of this section we need the following lemma.

Lemma 1 ([8, p. 386, Theorem 1.1]). The following series transformation formula holds

$$
\sum_{n=0}^{\infty} a_{n} H_{n}(x) \frac{t^{n}}{n !}=e^{2 x t-t^{2}} \sum_{n=0}^{\infty}(-1)^{n} H_{n}(x-t) \frac{t^{n}}{n !}\left\{\sum_{k=0}^{n}\left(\begin{array}{l}
n \\
k
\end{array}\right)(-1)^{k} a_{k}\right\},
$$

where

$$
f(t)=\sum_{k=0}^{\infty} a_{k} t^{k}
$$

is an arbitrary function, analytical in a neighborhood of zero. 
Theorem 7. Let $A$ be a matrix in $\mathbb{C}^{r \times r}$ such that $\operatorname{Re}(z)>0$ for all $z \in \sigma(A)$. Then Gegenbauer matrix polynomials have the series transformation formula

$$
\sum_{n=0}^{\infty} a_{n} C_{n}^{A}(x) z^{n}=\rho^{-A} \sum_{n=0}^{\infty}(-1)^{n} C_{n}^{A}\left(\frac{x-z}{\rho}, \frac{1}{\rho}\right) z^{n}\left\{\sum_{k=0}^{n}\left(\begin{array}{l}
n \\
k
\end{array}\right)(-1)^{k} a_{k}\right\},
$$

where $a_{k}$ are the Maclauren coefficients of an arbitrary function $f(t), \rho=1-2 x z+$ $y z^{2}$ and $|1-\rho|<1$.

Proof. For $c=1$ and $m=2,(3.7)$ reduce to

$$
C_{n}^{A}(x)=\frac{\Gamma^{-1}(A)}{n !} \int_{0}^{\infty} e^{-t} t^{A+\left(\frac{n}{2}-1\right) I} H_{n}(x \sqrt{t}) d t .
$$

Multiplying both hand sides of the above equation by $a_{n} z^{n}$ and summing over $n$ from zero to infinity, we get

$$
\sum_{n=0}^{\infty} a_{n} C_{n}^{A}(x) z^{n}=\sum_{n=0}^{\infty}(-1)^{n} C_{n}^{A}(x-z, \rho) z^{n}\left\{\sum_{k=0}^{n}\left(\begin{array}{l}
n \\
k
\end{array}\right)(-1)^{k} a_{k}\right\},
$$

Using this equation and

$$
C_{n}^{A}(x, c)=c^{-A} C_{n}^{A}\left(\frac{x}{c}, \frac{1}{c}\right),
$$

for $c=1-2 x z+y z^{2}$, we get the proof.

Note that $C_{n}^{A}\left(\frac{x-z}{\rho}, \frac{1}{\rho}\right)$ are Gegenbauer matrix polynomials with two-variables.

Our first application is the following.

Corollary 3. Let $B \in \mathbb{C}^{r \times r}$ and $C$ be matrix in $\mathbb{C}^{r \times r}$ such that $C+k I$ is invertible for all integer $k \geq 0$. Then we obtain bilateral series with hypergeometric matrix functions and Gegenbauer matrix polynomials

$$
\begin{aligned}
\sum_{n=0}^{\infty} C_{n}^{A}(x)(B)_{n}\left[(C)_{n}\right]^{-1}(z y)^{n} & \\
& =\rho^{-A} \sum_{n=0}^{\infty}(-1)^{n} C_{n}^{A}\left(\frac{x-z}{\rho}, \frac{1}{\rho}\right) F(-n I, B ; C ; y) z^{n} .
\end{aligned}
$$

Proof. Hypergeometric matrix functions have the representation [21]

$$
F(A, B ; C ; y)=\sum_{k \geq 0} \frac{(A)_{k}(B)_{k}\left[(C)_{k}\right]^{-1}}{k !} y^{k} \quad|y|<1,
$$


where $A, B, C$ are matrices in $\mathbb{C}^{r \times r}$ such that $C+k I$ is invertible for all integer $k \geq 0$. Setting $A=-n I$, where $n$ is a natural number, we get

$$
F(-n I, B ; C ; y)=\sum_{k=0}^{n}\left(\begin{array}{l}
n \\
k
\end{array}\right)(-1)^{k}(B)_{k}\left[(C)_{k}\right]^{-1} y^{k}
$$

Applying (4.3) with $a_{k}=(B)_{k}\left[(C)_{k}\right]^{-1} y^{k}$ we obtain (4.4).

Now, we can obtain bilateral generating function with hypergeometric matrix functions and Gegenbauer matrix polynomials with two-variables. Taking $C=2 A$ in (4.4) and using the generating function [5]

$$
\sum_{n=0}^{\infty}(B)_{n}\left[(2 A)_{n}\right]^{-1} C_{n}^{A}(x) r^{n}=(1-x r)^{-B} F\left(\frac{B}{2}, \frac{B}{2}+\frac{I}{2} ; A+\frac{I}{2} ; \frac{\left(x^{2}-1\right) r^{2}}{(1-x r)^{2}}\right),
$$

we have

$$
\begin{aligned}
\sum_{n=0}^{\infty}(-1)^{n} F & (-n I, B ; 2 A ; y) C_{n}^{A}\left(\frac{x-z}{\rho}, \frac{1}{\rho}\right) z^{n} \\
= & (1-x y z)^{-B} \exp (A \ln \rho) F\left(\frac{B}{2}, \frac{B}{2}+\frac{I}{2} ; A+\frac{I}{2} ; \frac{\left(x^{2}-1\right)(y z)^{2}}{(1-x y z)^{2}}\right),
\end{aligned}
$$

where $A B=B A$.

For the next corollary we use the binomial transform

$$
\sum_{k=0}^{n}\left(\begin{array}{l}
n \\
k
\end{array}\right)(-1)^{k}\left(\begin{array}{c}
p+k \\
k
\end{array}\right)=(-1)^{n}\left(\begin{array}{l}
p \\
n
\end{array}\right)
$$

which is a version of the Vandermonde convolution formula (see [33]). Here, $p$ is any complex number. The generating function for $a_{k}=\left(\begin{array}{c}p+k \\ k\end{array}\right)$ is

$$
(1-t)^{-p-1}=\sum_{k=0}^{\infty}\left(\begin{array}{c}
p+k \\
k
\end{array}\right) t^{k}
$$

According to (4.3) we obtain the following corollary.

Corollary 4. For any complex number $p$

$$
\sum_{n=0}^{\infty}\left(\begin{array}{c}
p+n \\
n
\end{array}\right) C_{n}^{A}(x) z^{n}=\rho^{-A} \sum_{n=0}^{\infty}\left(\begin{array}{l}
p \\
n
\end{array}\right) C_{n}^{A}\left(\frac{x-z}{\rho}, \frac{1}{\rho}\right) z^{n} .
$$

It is interesting that when $p$ is a positive integer, the right-hand side is finite. So, we have the closed form evaluation

$$
\sum_{n=0}^{\infty}\left(\begin{array}{c}
p+n \\
n
\end{array}\right) C_{n}^{A}(x) z^{n}=\rho^{-A} \sum_{n=0}^{p}\left(\begin{array}{l}
p \\
n
\end{array}\right) C_{n}^{A}\left(\frac{x-z}{\rho}, \frac{1}{\rho}\right) z^{n}
$$


We have the equation for Stirling numbers of the second kind extended for complex argument [9]

$$
(-1)^{n} n !\left\{\begin{array}{l}
\alpha \\
n
\end{array}\right\}=\sum_{k=0}^{n}\left(\begin{array}{l}
n \\
k
\end{array}\right)(-1)^{k} k^{\alpha},
$$

where $0 \neq \alpha \in \mathbb{C}$. Using this equation with $a_{k}=k^{\alpha}$ we have the following corollary.

Corollary 5. For any complex number $\alpha \neq 0$

$$
\sum_{n=0}^{\infty} n^{\alpha} C_{n}^{A}(x) z^{n}=\rho^{-A} \sum_{n=0}^{\infty}\left\{\begin{array}{l}
\alpha \\
n
\end{array}\right\} n ! C_{n}^{A}\left(\frac{x-z}{\rho}, \frac{1}{\rho}\right) z^{n} .
$$

Setting $\alpha=m \in \mathbb{Z} \cup\{0\}$ we have $\left\{\begin{array}{l}m \\ n\end{array}\right\}=0$ for $m<n$. Therefore, we obtain the closed form evaluation

$$
\sum_{n=0}^{\infty} n^{\alpha} C_{n}^{A}(x) z^{n}=\rho^{-A} \sum_{n=0}^{m}\left\{\begin{array}{c}
m \\
n
\end{array}\right\} n ! C_{n}^{A}\left(\frac{x-z}{\rho}, \frac{1}{\rho}\right) z^{n} .
$$

We note that taking $A=[\gamma]_{1 \times 1}$ the expression (4.6) coincides with the formula which was given by Srivastava ([34]) for the classical Gegenbauer polynomials by different means.

\section{ACKNOWLEDGEMENTS}

The present investigation was supported by the Scientific Research Project Administration of Akdeniz University.

\section{REFERENCES}

[1] R. Aktaş, "A new multivariable extension of humbert matrix polynomials," ser. AIP Conference Proceedings, vol. 1558, 2013, pp. 1128-1131.

[2] R. Aktaş, "A note on multivariable humbert matrix polynomials," Gazi University Journal of Science, vol. 27, no. 2, pp. 747-754, 2014.

[3] R. Aktaş, B. Çekim, and R. Şahin, "The matrix version for the multivariable humbert polynomials," Miskolc Mathematical Notes, vol. 13, no. 2, pp. 197-208, 2012.

[4] A. Altın and B. Çekim, "Some properties associated with hermite matrix polynomials," Utilitas Mathematica, vol. 88, pp. 171-181, 2012.

[5] A. Altın and B. Çekim, "Some miscellaneous properties for gegenbauer matrix polynomials," Utilitas Mathematica, vol. 92, pp. 377-387, 2013.

[6] A. Altın and Çekim B., "Generating matrix functions for chebyshev matrix polynomials of the second kind," Hacettepe Journal of Mathematics and Statistics, vol. 41, no. 1, pp. 25-32, 2012.

[7] R. S. Batahan, "A new extension of hermite matrix polynomials and its applications," Linear Algebra and its Applications, vol. 419, pp. 82-92, 2006.

[8] K. N. Boyadzhiev and A. Dil, "Series with hermite polynomials and applications," Publicationes Mathematicae Debrecen, vol. 80, no. 3-4, pp. 385-404, 2012.

[9] P. L. Butzer, A. A. Kilbas, and J. J. Trujillo, "Stirling functions of the second kind in the setting of difference and fractional calculus," Numerical Functinal Analysis and Optimization, vol. 24, no. 7-8, pp. 673-711, 2003. 
[10] B. Çekim, A. Altın, and R. Aktaş, "Some relations satisfied by orthogonal matrix polynomials," Hacettepe Journal of Mathematics and Statistics, vol. 40, no. 2, pp. 241-253, 2011.

[11] B. Çekim, A. Altın, and R. Aktaş, "Some new results for jacobi matrix polynomials," Filomat, vol. 27, no. 4, pp. 713-719, 2013.

[12] E. Defez and L. Jódar, "Chebyshev matrix polynomials and second order matrix differential equations," Utilitas Mathematica, vol. 61, pp. 107-123, 2002.

[13] E. Defez and L. Jódar, "Jacobi matrix differential equation, polynomial solutions,and their properties," Computers and Mathematics with Applications, vol. 48, pp. 789-803, 2004.

[14] V. A. Ditkin and A. Prudnikov, Integral Transforms and Operational Calculus. Oxford: Pergamon-Press, 1965.

[15] N. Dunford and J. Schwartz, Linear Operators. New York: Interscience, 1963, vol. I.

[16] H. Gould, "Inverse series relations and other expansions involving humbert polynomials," Duke Mathematical Journal, vol. 32, no. 4, pp. 697-712, 1965.

[17] H. Gould and A. Hopper, "Operational formulas connected with two generalizations of hermite polynomials," Duke Mathematical Journal, vol. 29, pp. 51-63, 1962.

[18] L. Jódar and R. Company, "Hermite matrix polynomials and second order matrix differential equations," Journal of Approximation Theory and its Applications, vol. 12, no. 2, pp. 20-30, 1996.

[19] L. Jódar, R. Company, and P. E., "Orthogonal matrix polynomials and systems of second order differential equations," Differential Equations and Dynamical Systems, vol. 3, no. 3, pp. 269-288, 1995.

[20] L. Jódar, R. Company, and E. Navarro, "Laguerre matrix polynomials and systems of second order differential equations," Applied Numerical Mathematics, vol. 15, pp. 53-63, 1994.

[21] L. Jódar and J. Cortes, "On the hypergeometric matrix function," Journal of Computational and Applied Mathematics, vol. 99, pp. 205-217, 1998.

[22] L. Jódar and J. Cortes, "Some properties of gamma and beta functions," Applied Mathematic Letters, vol. 11, no. 1, pp. 89-93, 1998.

[23] L. Jódar and J. Sastre, "On the laguerre matrix polynomials," Utilitas Mathematica, vol. 53, pp. 37-48, 1998.

[24] L. Kargin and V. Kurt, "Chebyshev-type matrix polynomials and integral transforms," Hacettepe Journal of Mathematics and Statistics (accepted).

[25] L. Kargin and V. Kurt, "Some relations on hermite matrix polynomials," Mathematical and Computational Applications, vol. 18, no. 3, pp. 323-329, 2013.

[26] G. Khammash, "Some bilateral generating relations involving gegenbauer matrix polynomials," Journal of Mathematical Sciences Advances and Applications, vol. 3, no. 1, pp. 89-100, 2009.

[27] G. Khammash and A. Shehata, "On humbert matrix polynomials," Asian Journal of Current Engineering and Maths, vol. 5, pp. 232-240, 2012.

[28] S. Khan and M. Al-Saad, "Summation formulae for gould-hopper generalized hermite polynomials," Computers and Mathematics with Applications, vol. 61, pp. 1536-1541, 2011.

[29] Z. M. G. Kishka, A. Shehata, and M. Abul-Dahab, "The generalized bessel matrix polynomials," Journal of Mathematical and Computational Science, vol. 2, pp. 305-316, 2012.

[30] M. Lahiri, "On a generalization of hermite polynomials," Proceedings of the American Mathematical Society, vol. 27, pp. 117-121, 1971.

[31] M. Metwally, "Operational rules and arbitrary order two-index two-variable hermite matrix generating functions," Acta Mathematica Academiae Paedagogicae Nyíregyháziensis, vol. 27, no. 1, pp. 41-49, 2011

[32] E. Rainville, Special Functions. New York: The Macmillan Company, 1960.

[33] J. Riordan, Combinatorial Identities. Huntington, New York: Robert E. Krieger Publishing Company, 1979. 
[34] H. Srivastava, "Some families of generating functions associated with the stirling numbers of the second kind," Journal of Mathematical Analysis and Applications, vol. 251, pp. 752-769, 2000.

[35] H. Srivastava and H. Manocha, A Treatise on Generating Functions. New York, Chichester, Brisbane, Toronto: Halsted Press-Ellis Horwood Limited-John Wiley and Sons, 1984.

[36] L. Upadhyaya and A. Shehata, "On legendre matrix polynomials and its applications," International Transactions in Mathematical Sciences \& Computer, vol. 4, no. 2, pp. 291-310, 2011.

[37] S. Varma, B. Çekim, and F. Taşdelen, "On konhauser matrix polynomials," Ars Combinatoria, vol. 100, pp. 193-204, 2011.

Authors' addresses

Levent Kargin

Akdeniz University, Akseki Vocational School, TR-07630 Antalya, Turkey

E-mail address: lkargin@akdeniz.edu.tr

\section{Veli Kurt}

Akdeniz University, Faculty of Science Department of Mathematics, TR-07058 Antalya, Turkey

E-mail address: vkurteakdeniz.edu.tr 